\title{
Metronomic Nanocaged Doxorubicin Prevents Chemoresistance and Cardiotoxicity in Breast Cancer
}

\author{
Serena Mazzucchelli ${ }^{1}$, Marta Truffi ${ }^{1}$, Michela Bellini ${ }^{3}$, Maria A. Rizzuto ${ }^{3}$, Davide Prosperi ${ }^{3}$, Fabio Corsi $^{1,2}$ \\ ${ }^{1}$ Department of Biomedical and Clinical Sciences, University of Milan \\ Via G. B. Grassi 74, 20157 Milan, Italy \\ serena.mazzucchelli@gmail.com; marta.truffi@unimi.it \\ ${ }^{2}$ Surgery Department, Breast Unit, ICS Maugeri S. p. A. SB \\ Pavia, Italy \\ fabio.corsi@unimi.it \\ ${ }^{3}$ Department of Biotechnology and Biosciences, University of Milan- Bicocca \\ Piazza della Scienza 2, 20126 Milan, Italy \\ michi.bellini@gmail.com; m.rizzuto3@campus.unimib.it; davide.prosperi@unimib.it
}

\section{Extended Abstract}

Low-dose metronomic (LDM) chemotherapy is based on frequent administration of a lower dose of drugs, without the need of extensive interruptions [1]. LDM affects the vasculature growth and repair [1] and stimulates the host immune system against the tumor [2], while it has not activity against tumor cells. Nanotechnology could improve the effectiveness of LDM chemotherapy, by taking advantage of the unique targeting efficiency of engineered nanocarriers [3].

In the present work, we propose a new concept of low dose "nanometronomic" (LDNM) chemotherapy, using doxorubicin (DOX) as pilot drug. As an ideal DOX nanocarrier, we used H-Ferritin (HFn) nanocages, owing to its affinity for transferrin receptor 1 (TfR-1), which is constitutively overexpressed in cancer cells [4]. HFn-DOX complex was recently demonstrated to overcome chemoresistance by actively promoting DOX nuclear translocation in vitro [4].

4T1-L tumor bearing mice were treated with placebo, DOX, liposomal-DOX (pl-DOX) or HFn-DOX under our LDNM setting. The progression of tumor volume was monitored in vivo demonstrating that HFn-DOX could decrease tumor growth, while DOX displayed a tumor progression similar to the control. An even better effect was achieved with pl-DOX, which was indeed able to arrest the tumor development. Moreover, we find that HFn-DOX antitumor effect is attributable to multiple nanodrug actions beyond cell killing, including inhibition of tumor angiogenesis and avoidance of chemoresistance. Otherwise, although an even better reduction of tumor progression was achieved with pl-DOX a fivefold increase in MDR1positive cells has been displayed, suggesting that liposomal DOX is not suitable in view of a protracted metronomic treatment, due to the onset of chemoresistance. [5]. Multiparametric assessment of heart tissues, has been performed to investigate the cardiotoxicity of the metronomic HFn-DOX treatment [6]. Histological evaluations of cardiomyocyte crosssections from mice treated with HFn-DOX, pl-DOX, DOX or non-treated was coupled with ultrastructural assessment of morphological alterations in mitochondria number, surface area and cristae depletion. Pathological alterations were detected in DOX and pl-DOX samples suggesting a strong cellular damage response compared to HFn-DOX samples. Therefore, the absence of obvious alterations in heart samples from mice treated with HFn-DOX strongly supports the lack of cardiotoxicity in LDNM HFn-DOX treatment, even compared to pl-DOX, which is currently considered the most safe anthracycline therapy in terms of cardiotoxicity. DOX and pl-DOX ultrastructural cardiac alterations were also associated to functional ones, such as the decrease in mitochodrial membrane potential and the reduction in the reactive oxygen species (ROS) quencher, GSH, confirming mitochondrial dysfunction induced by treatment with DOX and pl-DOX only.

In summary, this study provides robust evidence that LDNM monotherapy with HFn-DOX is expected to remodel the therapeutic outcome of advanced metastatic BC compared to the drug alone and also to improve anthracycline therapies based on liposomal DOX, with a redefinition of the central role of DOX for solid malignancies under the new perspective of metronomic treatments. 


\section{References}

[1] D. Loven, E. Hasnis, F. Bertolini, Y. Shaked, "Low-dose metronomic chemotherapy: from past experience to new paradigms in the treatment of cancer," Drug Discov Today, vol. 18, pp. 193-201, 2013.

[2] I. Kareva, D. J. Waxman, G. L. Klement, "Metronomic chemotherapy: an attractive alternative to maximum tolerated dose therapy that can activate anti-tumor immunity and minimize therapeutic resistance," Cancer Lett., vol. 358, pp. 100-106, 2015.

[3] S. S. W. Ng, A. Sparreboom, Y. Shaked, C. Lee, S. Man, N. Desai, P. Soon-Shiong, W. D. Figg, R. S. Kerbel, "Influence of formulation vehicle on metronomic taxane chemotherapy: albumin-bound versus cremophor EL-based paclitaxel," Clin Cancer Res., vol. 12, pp. 4331-4338, 2006.

[4] M. Bellini, S. Mazzucchelli, E. Galbiati, S. Sommaruga, L. Fiandra, M. Truff, M. A. Rizzuto, M. Colombo, P. Tortora, F. Corsi, D. Prosperi, "Protein nanocages for self-triggered nuclear delivery of DNA-targeted chemotherapeutics in cancer cells," J Controlled Rel., vol. 196, pp. 184-196, 2014.

[5] L. Bao, A. Haque, K. Jackson, S. Hazari, K. Moroz, R. Jetly, S. Dash, "Increased expression of P-glycoprotein is associated with doxorubicin chemoresistance in the metastatic 4T1 breast cancer model," Am J Pathol., vol. 178, pp. 838-852, 2011.

[6] S. Khiati, I. Dalla Rosa, C. Sourbier, X. Ma, V. A. Rao, L. M. Neckers, H. Zhang, Y. Pommier, "Mitochondrial topoisomerase I (Top1mt) is a novel limiting factor of doxorubicin cardiotoxicity," Clin Cancer Res., vol. 20, pp. 48734881, 2014. 